\title{
Resonances near the real axis imply existence of quasimodes
}

\author{
Plamen Stefanov \\ Department of Mathematics \\ East Carolina University \\ Greenville, NC 27858, USA
}

\begin{abstract}
We prove that if there exists a sequence of resonances converging rapidly to the real axis, then for any strictly compact set $K$ which interior contains the scatterer, one can find a sequence of real quasimodes with quasimode states supported in $K$.
\end{abstract}

Resumé. On prouve que s'il existe une suite de résonances qui convergent rapidement vers l'axe réel, alors pour chaque ensemble srictement convexe $K$ dont l'intérieur contient la perturbation compacte, on peut trouver une suite de quasimodes réels et des états associés ayant leurs supports dans $K$.

\section{Version française abrégée: L'existence de résonances près de l'axe réel implique l'existence de quasimodes}

Soit $P$ un opérateur auto-adjoint qui coïncide avec le Laplacien à l'extérieur d'un compact $K_{0} \subset \mathbf{R}^{n}, n \geq 2$. On se propose d'examiner la liaison entre les quasimodes réels à support compact et l'existence de résonances de $P$ convergeant rapidement vers l'axe réel. On va étudier le problème dans le cadre de la théorie abstraite de "black box" scattering [SjZ]. Il est connu (cf. [SV2], [TZ], [S]) que l'existence de quasimodes réels $q_{j} \rightarrow \infty$ implique l'existence de résonances $\lambda_{j}$ avec $0 \leq \operatorname{Im} \lambda_{j}=O\left(\left|\lambda_{j}\right|^{-\infty}\right)$. Dans cette note on prouve un résultat inverse c'est-à-dire s'il existe une suite de résonances qui convergent rapidement vers l'axe réel, alors on peut trouver une suite de quasimodes $q_{j} \rightarrow \infty$.

Theéorème 1 Soit $K \supset K_{0}$ un ensemble convexe compact. Supposons qu'il existe une suite $\lambda_{j}$ de résonances telles que $0 \leq \operatorname{Im} \lambda_{j}=O\left(\left|\lambda_{j}\right|^{-\infty}\right), 0<\operatorname{Re} \lambda_{j} \rightarrow \infty$. Alors il existe une suite de quasimodes $0<q_{j} \rightarrow \infty$ et de fonctions $v_{j}$, telles que $\operatorname{supp} v_{j} \subset K,\left\|v_{j}\right\|=1$ et $\left(P-q_{j}^{2}\right) v_{j}=O\left(q_{j}^{-\infty}\right), j=1,2, \ldots \infty$. 
Idée de la preuve: Un candidat naturel pour les quasimodes sont les fonctions associées aux résonances qu'on va tronquer au-delà de la frontière de $K$. Posons $q_{j}:=\operatorname{Re} \lambda_{j}$. Soit $K$ comme ci-dessus et posons $v_{j}:=\chi u_{j} /\left\|\chi u_{j}\right\|$, où $u_{j}$ est la solution sortante de $\left(P-\lambda_{j}^{2}\right) u_{j}=0$ et $\chi \in C_{0}^{\infty}\left(\mathbf{R}^{n}\right)$ est une fonction à support dans $K$ et telle que $\chi=1$ sur $K^{\prime}$ avec $K_{0} \subset \subset$ $K^{\prime} \subset \subset K$. On normalise $u_{j}$ de telle façon que $\left\|u_{j}\right\|_{L^{2}(K)}=1$. Le théorème découle de l'estimation suivante

$$
\left\|u_{j}\right\|_{H^{1}\left(K \backslash K^{\prime}\right)}=O\left(\left|\lambda_{j}\right|^{-\infty}\right)\left\|u_{j}\right\|_{L^{2}(K)} .
$$

Nous allons travailler avec des opérateurs pseudodifférentiels avec un large paramètre $\lambda \in \Lambda$ (cf. [G], [SV1], [SjV]). Supposons que $\Lambda:=\left\{\lambda_{j} ; j=1,2, \ldots\right\}$. Pour démontrer (1), il est suffisant de montrer que $\widetilde{\mathrm{WF}}\left(\left.u\right|_{K \backslash K^{\prime}}\right)=\emptyset$. Cela découle de l'argument suivant. Sur $\partial K^{\prime}$ le front d'onde de la fonction $u=u(x, \lambda), \lambda \in \Lambda$ est inclus dans la région hyperbolique. Pour la justification de cela on examine le problème à bord pour $\left(P-\lambda^{2}\right) u=0$ avec données sur une autre frontière $\partial K^{\prime \prime}$ strictement convexe qui est située entre $\partial K_{0}$ et $\partial K^{\prime}$ et on applique les propriétés sortantes de la solution qui sont liées avec la paramétrix. La formule de Green implique

$$
\left(\left.\left(N^{*}(\lambda)-N(\lambda)\right) u\right|_{\partial K^{\prime}},\left.u\right|_{\partial K^{\prime}}\right)_{L^{2}\left(\partial K^{\prime}\right)}=\left(\lambda^{2}-\bar{\lambda}^{2}\right)\|u\|_{L^{2}\left(K^{\prime}\right)}^{2}=O\left(|\lambda|^{-\infty}\right) .
$$

Ici $N(\lambda)$ désigne l'opérateur de Neumann qui aux données de Dirichlet sur $\partial K^{\prime}$ associe les dérivées normales des solutions sortantes de $\left(P-\lambda^{2}\right) u=0$ sur $\partial K^{\prime}$. Il est suffissant d'étudier $N(\lambda)$ seulement dans la région hyperbolique. Alors $-i\left(N^{*}-N\right)$ est un opérateur pseudodifférentiel positif avec large paramètre ayant pour symbol principal $2 \lambda \sqrt{1-|\xi|_{x}^{2}}$. Cela implique $\left.u\right|_{\partial K^{\prime}}=O\left(|\lambda|^{-\infty}\right)$. Etant donné que $\widetilde{\mathrm{WF}}\left(\left.u\right|_{\partial K^{\prime}}\right)$ est dans la région hyperbolique, on obtient $\widetilde{\mathrm{WF}}\left(\left.u\right|_{\partial K^{\prime}}\right)=\emptyset$ et on en déduit facilement $(1)$.

There has been a considerable progress recently in understanding the relationship between resonances (scattering poles) near the real axis and existence of real quasimodes for compactly supported perturbations of the Laplacian. It was shown in [SV2] that existence of a sequence of real quasimodes $q_{j} \rightarrow \infty$ corresponding to quasimode states supported in a fixed compact set implies existence of resonances $\lambda_{j}$ with $0 \leq \operatorname{Im} \lambda_{j}=O\left(\left|\lambda_{j}\right|^{-\infty}\right)$. Moreover, quasimodes are $O\left(\left|\lambda_{j}\right|^{-\infty}\right)$ perturbations of resonances, counting multiplicities, and this allows us to obtain lower bounds on the asymptotic distribution of those resonances (see [TZ], $[\mathrm{S}]$ ). The purpose of this note is to prove a statement converse to that proved in [SV2] — if there exists a sequence of resonances $\lambda_{j}$ tending rapidly to the real axis, then one can find real quasimodes $q_{j} \rightarrow \infty$. A natural candidate for the quasimode states are the resonance states, indeed, cut-off near the boundary of some compact set. We are going to show that those resonance states are $O\left(\left|\lambda_{j}\right|^{-\infty}\right)$ in any compact set away from the convex hull of the scatterer, so after cutting them off we get quasimode states. Although our proof does not show how to construct those quasimodes states (assuming that resonance states are unknown), it suggests that in any situation where one wants to show existence of "almost real" resonances, constructing real quasimodes should always be possible. 
We work in the abstract black box scattering setting for compactly supported perturbations of the Laplacian. Let $K_{0} \subset \mathbf{R}^{n}, n \geq 2$, be a fixed compact set and let

$$
\mathcal{H}:=\mathcal{H}_{0} \oplus L^{2}\left(\mathbf{R}^{n} \backslash K_{0}\right)
$$

be a complex Hilbert space. Suppose that $P$ is a unbounded self-adjoint operator in $\mathcal{H}$ such that $D(P)$ projected on $L^{2}\left(\mathbf{R}^{n} \backslash K_{0}\right)$ is $H^{2}\left(\mathbf{R}^{n} \backslash K_{0}\right)$ and $P$ restricted to the same space coincides with $-\Delta$. Next, assume that $\mathbf{1}_{\mathcal{H}_{0}}(P+i)^{-1}$ is compact, where $\mathbf{1}$ stands for the projection operator. Then one can define resonances of $P$ by the method of complex scaling [SjZ]. They are also the poles of the meromorphic continuation of the cut-off resolvent $R_{\chi}(\lambda)=\chi\left(P-\lambda^{2}\right)^{-1} \chi$ from $\operatorname{Im} \lambda<0$ to a neighborhood of the real axis in $\mathbf{C}$, where $\chi$ is any smooth cut-off function equal to 1 in $K_{0}$. In what follows $M \subset \subset N$ (or $N \supset \supset M$ ) indicates that the closure of the set $M$ is contained in the interior of $N$.

Theorem 1 Let $K \supset \supset K_{0}$ be a convex compact set. Suppose that there exists a sequence $\lambda_{j}$ of resonances with $0 \leq \operatorname{Im} \lambda_{j}=O\left(\left|\lambda_{j}\right|^{-\infty}\right), 0<\operatorname{Re} \lambda_{j} \rightarrow \infty$. Then there exists a sequence of quasimodes $0<q_{j} \rightarrow \infty$ and quasimode states $v_{j}$, such that $\operatorname{supp} v_{j} \subset K,\left\|v_{j}\right\|=1$ and $\left(P-q_{j}^{2}\right) v_{j}=O\left(q_{j}^{-\infty}\right), j=1,2, \ldots \infty$.

Proof. It is well-known that existence of resonances $\lambda_{j}$ implies existence of resonance states $u_{j}$ such that $\left(P-\lambda_{j}^{2}\right) u_{j}=0$ and $u_{j} \neq 0$ are $\lambda_{j}$-outgoing. Let $K$ be as above and set $v_{j}:=\chi u_{j} /\left\|\chi u_{j}\right\|$, where $\chi$ is a smooth function supported in $K$ and $\chi=1$ on the strictly convex compact $K^{\prime}$ with smooth boundary, where $K_{0} \subset \subset K^{\prime} \subset \subset K$. (A more precise statement would be to say that $\chi=\operatorname{Id}_{\mathcal{H}_{0}} \oplus \tilde{\chi}$ where $\tilde{\chi}$ is the operator of multiplication by $\chi(x)$ in $L^{2}\left(\mathbf{R}^{n} \backslash K_{0}\right)$.) We also set $q_{j}:=\operatorname{Re} \lambda_{j}$. In order to complete the proof of the theorem, it suffices to show that

$$
\left\|u_{j}\right\|_{H^{1}\left(K \backslash K^{\prime}\right)}=O\left(\left|\lambda_{j}\right|^{-\infty}\right)\left\|u_{j}\right\|_{L^{2}(K)} .
$$

Here and below we are going to use the notation $L^{2}(U)$, where $K_{0} \subset U$ to denote $\mathcal{H}_{0} \oplus$ $L^{2}\left(U \backslash K_{0}\right)$, etc.

Denote $\Lambda:=\left\{\lambda_{j} ; j=1,2, \ldots\right\}$. In what follows we are always going to assume that

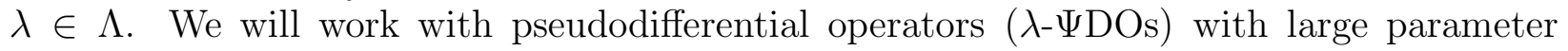
$\lambda \in \Lambda$ (see [G], [SV1], [SjV]). Set $u=u(x, \lambda):=u_{j}, \lambda=\lambda_{j} \in \Lambda$. Let us normalize $u$ so that $\|u\|_{L^{2}(K)}=1$. Then (2) would be a consequence of

$$
\widetilde{\mathrm{WF}}\left(\left.u\right|_{K \backslash K^{\prime}}\right)=\emptyset .
$$

To prove (3), choose another strictly convex set $K^{\prime \prime}$ with smooth boundary, such that $K_{0} \subset \subset K^{\prime \prime} \subset \subset K^{\prime}$ and let us study $f:=\left.u\right|_{\partial K^{\prime \prime}}$. The compactification $\widetilde{T^{*}}\left(\partial K^{\prime \prime}\right) \cong T^{*}\left(\partial K^{\prime \prime}\right) \cup$ $S^{*}\left(\partial K^{\prime \prime}\right)$ of the cotangent bundle $T^{*}\left(\partial K^{\prime \prime}\right)$ can be naturally decomposed into a hyperbolic region $|\xi|_{x}<1-\varepsilon$, a glancing region $\left.|| \xi\right|_{x}-1 \mid<\varepsilon$ and an elliptic region $|\xi|_{x}>1+\varepsilon$ (including the infinite points) with some $0<\varepsilon \ll 1$. Near $\partial K^{\prime \prime}$ (and in the exterior of $K^{\prime \prime}$ ), 
$u$ is the outgoing solution to the problem $\left(-\Delta-\lambda^{2}\right) u=0, u=f$ on $\partial K^{\prime \prime}$. Thus one can construct a parametrix for $u$ as in [G], [SV1]. Using this, we get for $\tilde{u}:=u /\|u\|_{L^{2}\left(\partial K^{\prime \prime}\right)}$ that

$$
\begin{aligned}
\widetilde{\mathrm{WF}}\left(\left.\tilde{u}\right|_{K \backslash K^{\prime}}\right) \subset & \left\{(x, \xi) \in T^{*}\left(K \backslash K^{\prime}\right) ;|\xi|_{x}=1,(x, \xi)\right. \text { belongs to the outgoing ray } \\
& \text { issued from some } \left.(y, \eta) \in T^{*}\left(\partial K^{\prime \prime}\right)\right\}
\end{aligned}
$$

Since $u$ solves $-\Delta u=\lambda^{2} u$ near $\partial K^{\prime \prime}$, by standard elliptic estimates we get that the $H^{2}$-norm of $u$ in a small neighborhood of $\partial K^{\prime}$ is $O\left(|\lambda|^{2}\right)\|u\|_{L^{2}(K)}=O\left(|\lambda|^{2}\right)$. By the trace theorem, $\|u\|_{L^{2}\left(\partial K^{\prime \prime}\right)}=O\left(|\lambda|^{2}\right)$. Thus we can replace $\tilde{u}$ in (4) by $u$.

In particular, (4) implies that $\widetilde{\mathrm{WF}}\left(\left.u\right|_{\partial K^{\prime}}\right)$ is included entirely in the hyperbolic region for $\varepsilon$ small enough. Indeed, all outgoing rays issued from $T^{*}\left(\partial K^{\prime \prime}\right)$, even the glancing ones, will make a positive angle with $\partial K^{\prime}$, uniformly bounded from below, which proves our claim. In other words, the contribution from the elliptic region in $\partial K^{\prime \prime}$ is $O\left(|\lambda|^{-\infty}\right)$ small on $\partial K^{\prime}$, the contribution from the other two regions in $\partial K^{\prime \prime}$ is similar on $\partial K^{\prime}$ but with a shift to the left (smaller $\left|\xi_{x}\right|$ 's) and that shifts the wave front set to the hyperbolic region.

Let $\nu$ be the outer unit normal to $\partial K^{\prime}$ and denote by $N: f \mapsto \partial u /\left.\partial \nu\right|_{\partial K^{\prime}}$ the Neumann operator that maps the Dirichlet data $f$ on $\partial K^{\prime}$ to the normal derivative on $\partial K^{\prime}$ of the outgoing solution to $\left(P-\lambda^{2}\right) u=0$. By Green's formula,

$$
\left(\left.\left(N^{*}(\lambda)-N(\lambda)\right) u\right|_{\partial K^{\prime}},\left.u\right|_{\partial K^{\prime}}\right)_{L^{2}\left(\partial K^{\prime}\right)}=\left(\lambda^{2}-\bar{\lambda}^{2}\right)\|u\|_{L^{2}\left(K^{\prime}\right)}^{2}=O\left(|\lambda|^{-\infty}\right) .
$$

Green's formula in the black box setting can be justified by considering $\left(P \chi_{K^{\prime}} u, u\right)$, where $\chi_{K^{\prime}}$ is the characteristic function of $K^{\prime}$, and using the fact that $P$ is self-adjoint. Consider the self-adjoint operator $Q(\lambda):=i^{-1}\left(N^{*}(\lambda)-N(\lambda)\right)$. Since $\widetilde{\mathrm{WF}}\left(\left.u\right|_{\partial K^{\prime}}\right)$ is in the hyperbolic region, we can replace $N^{*}-N=i Q$ in (5) by the corresponding hyperbolic parametrix $i Q_{h}$. Hence we get (see e.g., [SV1]) that the principal symbol of $Q_{h}$ is $\sigma_{p}\left(Q_{h}\right)=2 \lambda \sqrt{1-|\xi|_{x}^{2}}$. Thus

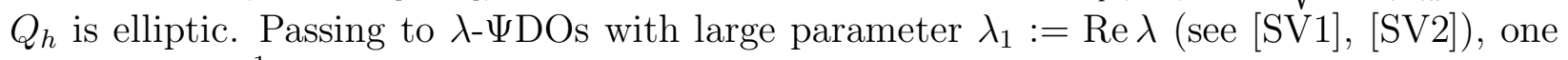
can extend $\lambda_{1}^{-1} Q_{h}$ to a strictly positive zero order operator in all regions. The square root $Q_{h}^{1 / 2}$ is an elliptic $\lambda$ - $\Psi$ DO again and (5) implies $Q_{h}^{1 / 2} u=O\left(|\lambda|^{-\infty}\right)$. Thus $\left.u\right|_{\partial K^{\prime}}=O\left(|\lambda|^{-\infty}\right)$ in $L^{2}\left(\partial K^{\prime}\right)$. Since $\widetilde{\mathrm{WF}}\left(\left.u\right|_{\partial K^{\prime}}\right)$ is in the hyperbolic region, we get the same for all (tangential) derivatives of $\left.u\right|_{\partial K^{\prime}}$, thus $\overline{\mathrm{WF}}\left(\left.u\right|_{\partial K^{\prime}}\right)=\emptyset$. Solving the problem $\left(-\Delta-\lambda^{2}\right) u=0,\left.u\right|_{\partial K^{\prime}}=\left.u\right|_{\partial K^{\prime}}$ outside $K^{\prime}$, we get (3) and this completes the proof of Theorem 1.

Remark. ${ }^{1}$ The arguments above based on the ellipticity of $Q$ can be replaced by Proposition 2.1 in [B] if $K$ is a ball, which also allows us to obtain exponentially small error terms in case of resonances converging exponentially fast to the real axis.

\section{References}

[B] N. Burq, Dcroissance de l'énergie locale de l'équation des ondes pour le problème extérieur et absence de résonance au voisinage du réel, Acta Math. 180(1998), no. 1, 1-29.

\footnotetext{
${ }^{1}$ due to M. Zworski
} 
[G] C. GÉRARD, Asymptotique des poles de la matrice de scattering pour deux obstacles strictement convex, Bull. Soc. Math. France, Mémoire n. 31, 116, 1988.

[SjV] J. Suöstrand And G. Vodev, Asymptotics of the number of Rayleigh resonances, Math. Ann. 309(1997), 287-306.

[SjZ] J. Sjöstrand AND M. Zworski, Complex scaling and the distribution of scattering poles, Journal of AMS 4(4)(1991), 729-769.

[S] P. Stefanov, Quasimodes and resonances: sharp lower bounds, Duke Math. J., to appear.

[SV1] P. Stefanov And G. Vodev, Distribution of resonances for the Neumann problem in linear elasticity outside a strictly convex body, Duke Math. J., 78(1995), 677-714.

[SV2] P. Stefanov And G. Vodev, Neumann resonances in linear elasticity for an arbitrary body, Comm. Math. Phys. 176(1996), 645-659.

[TZ] H.-S. TAng And M. Zworski, From quasimodes to resonances, Math. Res. Lett. 5(1988), 261-272. 